\title{
COMPARISON OF ZIEHL-NEELSEN STAINS WITH FINE NEEDLE ASPIRATION TECHNIQUE IN DIAGNOSIS OF TUBERCULOUS LYMPHADENITIS IN A TERTIARY CARE HOSPITAL, SOUTH BIHAR (INDIA)
}

\author{
Imtiaz Ahmad', Asim Mishra², Chandan Kumar Poddar', Pawan Kumar Chaudhary4
}

${ }_{1}^{1}$ Assistant Professor, Department of Pathology, Vardhman Institute of Medical Sciences, Pawapuri, Nalanda.

${ }^{2}$ Associate Professor, Department of Pathology, Anugrah Narayan Magadh Medical College, Gaya.

${ }^{3}$ Research Scholar, Department of Microbiology, Indira Gandhi Institute of Medical Sciences, Patna.

${ }^{4}$ Associate Professor and HOD, Department of Pathology, Vardhman Institute of Medical Sciences, Pawapuri, Nalanda.

ABSTRACT
BACKGROUND
Tuberculosis continues to be a major health problem in developing countries. Tuberculosis is one of the oldest diseases known to
affect humans and is caused by Mycobacterium tuberculosis. Lymphadenopathy is the most common presentation of
extrapulmonary tuberculosis. Tuberculous (TB) lymphadenitis is one of the common causes of lymphadenopathy. This study was
conducted to compare cytology, ZN staining and culture findings of clinically suspected tuberculous lymphadenitis cases.

\section{MATERIALS AND METHODS}

The study design is a descriptive study. Total 220 patients of lymphadenopathy referred to the Department of Pathology, Vardhman Institute of Medical Sciences, Pawapuri, Nalanda, Bihar and Associated Hospital of Bihar between March 2015 and May 2017 were included. FNAC was performed in all these patients and smears were prepared. Smears were stained with haematoxylin and eosin stain. ZN staining for acid-fast bacilli (AFB) was carried out on separate slide. Using solid culture (BACTEC) as the gold standard, we assessed the sensitivity, specificity, positive predictive value (PPV), negative predictive value (NPV) and Kappa value of the FNAC for detecting MTB and ZN staining for acid-fast bacilli (AFB) respectively.

\section{RESULTS}

Maximum number of patients [40\% (40/100)] were from age group of 11-30 years. Total 220 cases [55\% (55/100) females and $40 \%(40 / 100)$ males] were included in the study. Of 220 cases, $50(50 \%)$ had tuberculosis, 50 (50\%) had lymphadenitis other than tuberculosis, and $10(10 \%)$ had malignant lymphadenopathy, including $02(02 \%)$ cases of primary malignancy (i.e. lymphoma) and $9(09 \%)$ cases of metastasis to lymph nodes. The cytology suggestive of tuberculous lymphadenitis was found in 100 (45.45\%) cases out of total 220 cases. Ziehl-Neelsen stain demonstrated acid-fast bacilli (AFB) in 40 (18.18\%) cases and BACTEC isolated mycobacteria in $60(27.27 \%)$ cases. When setting the results of Culture (BACTEC) as the gold standard, the sensitivity, specificity, positive predictive value (PPV), negative predictive value (NPV) and Kappa value of the FNAC in the diagnosis of TB lymphadenitis, results were $96.15 \%, 96.00 \%, 95.23 \%$ and $96.77 \%$ and 0.9172 respectively. In addition, when compared with ZN stain in the diagnosis of TB lymphadenitis, the sensitivity, specificity, PPV, NPV and Kappa were 93.33\%, 98.88\%, 95.45\%, 98.34\% and 0.928 respectively.

\section{CONCLUSION}

FNAC is an assay that has high sensitivities, is optimally selected, efficient, easy to perform, and is an economical test for initial diagnostic workup in patients with TB lymphadenitis. Supplementation of ZN stain with FNAC increases the rates of diagnosis.

\section{KEYWORDS}

Tuberculosis, Lymphadenopathy, BACTEC, Fine Needle Aspiration Cytology, Ziehl-Neelsen Stain.

HOW TO CITE THIS ARTICLE: Ahmad I, Mishra A, Poddar CK, et al. Comparison of Ziehl-Neelsen stains with fine needle aspiration technique in diagnosis of tuberculous lymphadenitis in tertiary care hospital, South Bihar (India). J. Evolution Med. Dent. Sci. 2017;6(63):4598-4602, DOI: 10.14260/Jemds/2017/995

\section{BACKGROUND}

Tuberculosis (TB) is an infectious disease caused by various strains of Mycobacterium, mainly by Mycobacterium tuberculosis in humans, it has been present in humans since antiquity.[1] TB is a chronic, progressive infectious disease with a period of latency following initial infection. It occurs

Financial or Other, Competing Interest: None.

Submission 29-06-2017, Peer Review 25-07-2017,

Acceptance 31-07-2017, Published 07-08-2017.

Corresponding Author:

Dr. Chandan Kumar Poddar,

Research Scholar, Department of Microbiology,

Indira Gandhi Institute of Medical Sciences, Patna.

E-mail: chandan_microbiology@yahoo.co.in

DOI: $10.14260 /$ jemds $/ 2017 / 995$ productive cough, chest pain, and dyspnoea. Tuberculosis is the leading infectious cause of morbidity and mortality in adults worldwide, killing about 2 million people every year.[2]

India has the highest burden of tuberculosis in the world as reflected by the World Health Organization statistics for 2011, giving an estimated incidence of 2.2 million cases of tuberculosis for India out of a global incidence of 8.7 million cases. ${ }^{[3]}$

Tuberculosis continues to be a major health problem in developing countries. Tuberculosis is one of the oldest diseases known to affect humans and is caused by Mycobacterium tuberculosis. Lymphadenopathy is the most common presentation of extrapulmonary tuberculosis.[4,5] Tuberculous lymphadenitis can be presumptively diagnosed 
morphologically on fine needle aspiration cytology of lymph node. Fine needle aspiration cytology is now widely utilised as a first line diagnostic procedure in the diagnosis of palpable masses, including peripheral lymphadenopathy. Its value in the diagnosis of mycobacterial lymphadenitis in adults is well documented.

In extrapulmonary tuberculosis, the most common presentation is cervical lymphadenopathy, especially among the Asian population.[6,7] Lymph node enlargement could be due to tuberculosis, other inflammatory disease or fungal infection, or some underlying malignancy.[8] In general, tuberculous (TB) lymphadenitis is diagnosed using conventional methods such as histopathology on the basis of caseous necrosis and granuloma formation. The chances of acid-fast bacilli (AFB) identification in tissue section are less because xylene and formalin affect the sensitivity of ZiehlNeelsen (ZN) method to detect Mycobacterium tuberculosis in histopathology sections. ${ }^{[9]}$

Fine needle aspiration cytology (FNAC) is a simple, quicker, reliable, minimally invasive, and relatively cheap diagnostic modality with minimal risk of complications.[10] The efficacy of FNAC to diagnose TB lymphadenitis is directly proportional to presence of purulent material in sample.[11] AFB are commonly seen in purulent samples, which may not contain granuloma, caseous necrosis, or epithelioid cells. In the absence of ZN staining, sample can be wrongly diagnosed as acute suppurative lymphadenitis.[5]

Mycobacteria are slow growing and hence culture is not done routinely in all laboratories. Few studies have tried to correlate the cytological finding with microbiological results for the presence of acid-fast bacilli in smears and culture for Mycobacteria. The current study was conducted with following objectives.

The aims and objectives of this prospective study were (1) to describe presentation pattern of TB lymphadenitis and

(2) to compare the results of Culture (BACTEC), FNAC and ZN stain in the diagnosis of TB lymphadenitis.

\section{MATERIALS AND METHODS}

Study Design: A descriptive study. All tuberculous lymphadenitis isolates from sputum samples of clinically suspected cases of tuberculosis seen in Vardhman Institute of Medical Sciences, Pawapuri, Nalanda, Bihar and Associated Hospital of Bihar between March 2015 and May 2017 were included in the study. The study protocol was approved by the hospital ethics committee.

\section{Inclusion Criteria}

Patients attending the Department of Pathology, Vardhman Institute of Medical Sciences, Pawapuri, Nalanda, Bihar and Associated Hospital of Bihar, between March 2015 and May 2017, having fever, night sweats, cough for more than 3 weeks with sputum, loss of appetite, loss of weight, chest pain, haemoptysis and/or radiological evidence of tuberculosis were included.

\section{Sample Collection}

A total of 220 Fine needle aspirates were obtained from patients who presented with signs and symptoms of Tuberculosis after consent was given. Early morning sputum samples were collected in clean, sterile, leak proof, wide mouth containers.

\section{Sample Processing}

1. FNA material from lymph nodes was applied to prior labelled slides directly and direct smears prepared for ZN staining.

2. Leftover material in the hub of the needle was rinsed in one millilitre $(\mathrm{mL})$ normal saline and transferred into Bijou bottles. To this, one $\mathrm{mL}$ of $3.5 \% \mathrm{NaOCl}$ was added and the mixture incubated at room temperature for 15 minutes while shaking at regular intervals. One half of 1 $\mathrm{mL}$ of xylene was added and the mixture let to stand for 15 minutes undisturbed to float the bacilli. The creamy layer was carefully scooped using a wire loop and smears prepared on prior labelled, albumin-coated microscope slides. The slides were air-dried, heat fixed and stained by the ZN method. Remaining material was inoculated in BACTEC (Middlebrook 7H12B) vial taking care to have at least $0.5 \mathrm{~mL}$ volume of test material in the vial.

\section{Direct ZN}

FNA material obtained was smeared on slides and directly stained with strong Carbol fuchsine and steamed for five minutes. The slides were then washed with tap water and decolourised with 1\% acid alcohol until clear. Subsequent rinsing in tap water followed. Counter staining was done in Methylene blue for three minutes and finally rinsed in tap water and left to air dry. Examination was done using high power (X100) and reported using WHO format.

Total 220 clinically diagnosed patients of lymphadenopathy referred to the Department of Pathology, Vardhman Institute of Medical Sciences, Pawapuri, Nalanda, Bihar, between March 2015 and May 2017 were included in this study. The variables included in the study were age, sex, and site of lesion. Relevant history and examination of nodes were recorded.

Cytology smears and ZN stain smears were examined in Department of Pathology, Vardhman Institute of Medical Sciences, Pawapuri, Nalanda, Bihar. BACTEC vial containing aspirated material was sent to Central Pathology Laboratory, ANMMC, Gaya without delay where first medium was supplemented with mixture of antimicrobials called PANTA, which contains polymyxin $-\mathrm{B}$, amphotericin-B, nalidixic acid, trimethoprim and azlocillin to reduce the contamination. Then initial reading Growth Index (GI) was taken and then incubated at $37^{\circ} \mathrm{C}$. Readings of GI were taken on day $1,3,5,7$, 9, 12 for first 15 days and then weekly up to 45 days.

Nodes were aspirated after all aseptic measures with sterile disposable 23-G needle attached with $10 \mathrm{cc}$ disposable syringe. Multiple smears were prepared with part of aspirated material; two to three smears were stained with haematoxylin and eosin (H\&E) stain and ZN staining was performed on separate slide. All data were grouped and analysed. Smears stained with $H \& E$ stain (Figure 1: Granuloma formation, H\&E stain, 40 X.) were examined under microscope for the presence of granuloma, necrosis, Langhans giant cells, plasma cells, lymphocytes, macrophages, and neutrophils. Smears stained with ZN stain (Figure 2: Acid-fast bacilli in ZN stain, $100 \mathrm{X}$.) were examined under oil immersion objective for AFB.

Presence of sheets of epithelioid cells with lymphocytes and plasma cells with or without multinucleated giant cells were diagnosed as granulomatous lymphadenitis, and 
eosinophilic granular material containing inflammatory cells and necrotic cell debris was defined as caseous necrosis.[11] The TB abscess was described as degenerate caseous necrosis and/or liquefied necrotic material with marked degenerating and viable inflammatory cell infiltration without epithelioid granuloma.[12]

Data was recorded and statistically analysed and using solid culture (BACTEC) as the gold standard, we assessed the sensitivity, specificity, positive predictive value (PPV), negative predictive value (NPV) and Kappa value of the FNAC for detecting MTB and ZN staining for acid-fast bacilli (AFB) respectively.

\section{Statistical Analysis}

Statistical analysis was performed with commercially available software (SPSS 16.0; Inc.).

\section{RESULTS}

A total of 220 fine-needle aspirated specimens from lymph nodes were included in the study. Out of 220 cases, 100 aspirates were reported as cytomorphology suggestive of tuberculous lymphadenitis. The age ranged from 1 to 70 years, with the mean age of 35.5 years. Female preponderance was noted accounting for 55\% (55/100) of cases (Table 1). $40 \%(40 / 100)$ of the cases with suggestive cytomorphology of tubercular lymphadenitis were in the range of 11-30 years of age. Depending upon cytomorphological features, cases of granuloma 80 (80\%), necrosis $70(70 \%)$, acute inflammation 25 (25\%), lymphoid background 21 (21\%), giant cell 15 (15\%) were found in tuberculous lymphadenitis (Table 3 ). In present study, the most common site of involved lymph nodes was of the cervical region in $75 \%(75 / 100)$ of the cases (Table 2$)$.

TB lymphadenitis was found in 50 (50\%) cases, inflammatory lymphadenitis other than tuberculosis in 50 (50\%), and malignant lymphadenopathy in remaining 10 (10\%) cases, consisting $2(02 \%)$ cases of primary malignancy (i.e., lymphoma) and 9 (09\%) of metastasis to lymph node [Table 4]. Of 100 cases of lymphadenitis, ZN stain was found to be positive for AFB in 42 (42\%) cases [Table 5].

The cytology suggestive of tuberculous lymphadenitis was found in 100 (45.45\%) cases out of total 220 cases. ZiehlNeelsen stain demonstrated acid-fast bacilli (AFB) in 40 $(18.18 \%)$ cases and BACTEC isolated Mycobacteria in 60 (27.27\%) cases [Table 6].

When setting the results of Culture (BACTEC) as the gold standard, the sensitivity, specificity, positive predictive value (PPV), negative predictive value (NPV) and Kappa value the of FNAC in the diagnosis of TB lymphadenitis, results were $96.15 \%, 96.00 \%, \quad 95.23 \%$ and $96.77 \%$ and 0.9172 respectively. In addition, when compared with ZN stain in the diagnosis of TB lymphadenitis results, the sensitivity, specificity, PPV, NPV and Kappa of were 93.33\%, 98.88\%, $95.45 \%, 98.34 \%$ and 0.928 respectively.

\begin{tabular}{|c|c|c|c|c|}
\hline Age (years) & Male & Female & Total & Percentage \\
\hline $0-10$ & 4 & 6 & 10 & 10 \\
\hline $11-20$ & 8 & 12 & 20 & 20 \\
\hline $21-30$ & 9 & 11 & 20 & 20 \\
\hline $31-40$ & 8 & 7 & 15 & 15 \\
\hline $41-50$ & 7 & 3 & 10 & 10 \\
\hline $51-60$ & 4 & 6 & 10 & 10 \\
\hline
\end{tabular}

\begin{tabular}{|c|c|c|c|c|}
\hline $61-70$ & 4 & 6 & 10 & 10 \\
\hline More than 70 & 1 & 4 & 5 & 5 \\
\hline Total & $\mathbf{4 5}$ & $\mathbf{5 5}$ & $\mathbf{1 0 0}$ & $\mathbf{1 0 0}$ \\
\hline Percentage & 45 & 55 & 100 & 100 \\
\hline \multicolumn{6}{|c|}{ Table 1. Age and Sex-wise Distribution } \\
of Cases of Lymphadenopathy
\end{tabular}

\begin{tabular}{|c|c|}
\hline Site & Number (\%) \\
\hline Cervical lymph node & $75(75 \%)$ \\
\hline Axillary lymph node & $6(6 \%)$ \\
\hline Supraclavicular lymph node & $4(4 \%)$ \\
\hline Submandibular & $1(1 \%)$ \\
\hline Submental & $6(6 \%)$ \\
\hline Chest & $2(2 \%)$ \\
\hline Inguinal lymph node & $1(1 \%)$ \\
\hline Other & $3(3 \%)$ \\
\hline Total & $\mathbf{1 0 0}$ \\
\hline Table 2. Site Distribution of Tubercular Lymph Node \\
\hline
\end{tabular}

\begin{tabular}{|c|c|c|}
\hline Cytomorphological Patterns & Number & Percentage \\
\hline Granuloma & 80 & $80 \%$ \\
\hline Necrosis & 70 & $70 \%$ \\
\hline Lymphoid background & 25 & $25 \%$ \\
\hline Acute inflammation & 25 & $25 \%$ \\
\hline Giant cell & 15 & $15 \%$ \\
\hline Not documented & 5 & $5 \%$ \\
\hline \multicolumn{2}{|c|}{ Table 3. Cytomorphological Patterns } \\
\hline
\end{tabular}

\begin{tabular}{|c|c|c|c|}
\hline Type of Lesion & $\begin{array}{c}\text { Cytomorphological } \\
\text { Diagnosis }\end{array}$ & $\begin{array}{l}\text { No. of } \\
\text { Cases }\end{array}$ & $\%$ \\
\hline \multirow[t]{2}{*}{$\begin{array}{l}\text { Non-neoplastic } \\
\text { (inflammatory } \\
\text { lymphadenitis ) }\end{array}$} & $\begin{array}{l}\text { Tuberculous } \\
\text { lymphadenitis }\end{array}$ & 50 & 50 \\
\hline & $\begin{array}{c}\text { Chronic nonspecific } \\
\text { lymphadenitis }\end{array}$ & 29 & 29 \\
\hline Acute lymphadenitis & & 10 & 10 \\
\hline \multirow[t]{2}{*}{$\begin{array}{l}\text { Neoplastic (malignant } \\
\text { lymphadenopathy) }\end{array}$} & $\begin{array}{l}\text { Metastasis to } \\
\text { lymph node }\end{array}$ & 9 & 9 \\
\hline & $\begin{array}{l}\text { Hodgkin's } \\
\text { lymphoma }\end{array}$ & 1 & 1 \\
\hline $\begin{array}{l}\text { Non-Hodgkin's } \\
\text { lymphoma }\end{array}$ & & 1 & 1 \\
\hline Total & & 100 & 100 \\
\hline
\end{tabular}

\begin{tabular}{|c|c|c|c|c|}
\hline $\begin{array}{l}\text { Cytomorphological } \\
\text { Picture }\end{array}$ & $\begin{array}{c}\text { AFB } \\
\text { Positive } \\
\text { Cases }\end{array}$ & \begin{tabular}{|c|} 
AFB \\
Negative \\
Cases \\
\end{tabular} & Total & $\%$ \\
\hline $\begin{array}{l}\text { Epithelioid granuloma } \\
\text { with caseous necrosis }\end{array}$ & 20 & 17 & 37 & 37 \\
\hline $\begin{array}{l}\text { Necrosis only without } \\
\text { inflammatory cells }\end{array}$ & 05 & 00 & 05 & 05 \\
\hline $\begin{array}{l}\text { Necrosis with } \\
\text { polymorphs }\end{array}$ & 17 & 10 & 27 & 27 \\
\hline $\begin{array}{l}\text { Neither necrosis nor } \\
\text { granuloma }\end{array}$ & 00 & 31 & 31 & 31 \\
\hline Total & 42 & 58 & 100 & 100 \\
\hline Percentage & 42 & 58 & 100 & 100 \\
\hline \multicolumn{5}{|c|}{$\begin{array}{c}\text { Table 5. AFB Positivity in Various Cytomorphological Sub- } \\
\text { patterns in Cases of Lymphadenitis }\end{array}$} \\
\hline
\end{tabular}




\begin{tabular}{|c|c|c|}
\hline Cases & No. & Percentage \\
\hline $\begin{array}{l}\text { Cytology suggestive of } \\
\text { tuberculous lymphadenitis }\end{array}$ & 100 & 45.45 \\
\hline ZN staining demonstrating AFB & 40 & 18.18 \\
\hline Culture isolating mycobacteria & 60 & 27.27 \\
\hline Total Cases & 220 & \\
\hline \multicolumn{3}{|c|}{$\begin{array}{l}\text { Table 6. Correlation between Cytology, } \\
\text { ZN Staining and Culture Findings }\end{array}$} \\
\hline
\end{tabular}

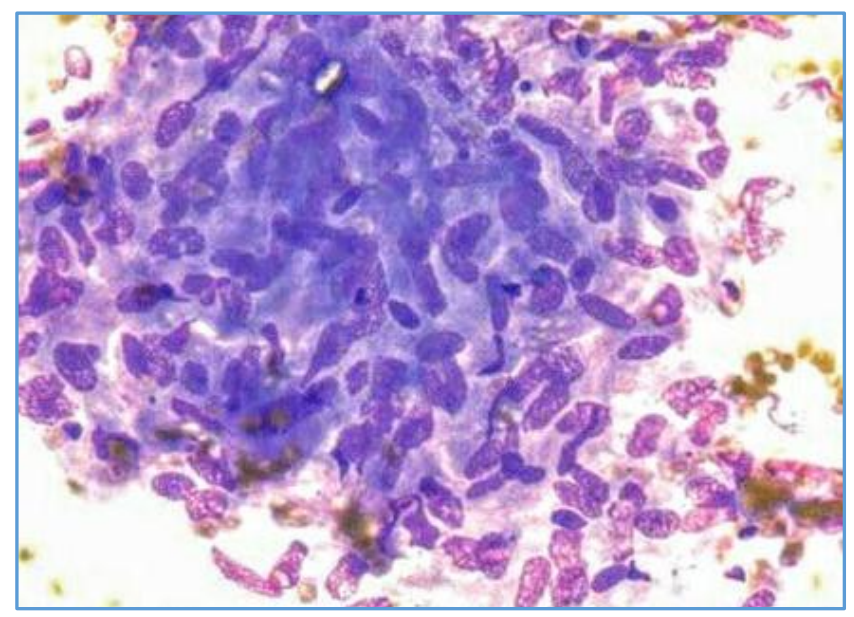

Figure 1. Granuloma Formation, H\&E Stain, $40 X$.

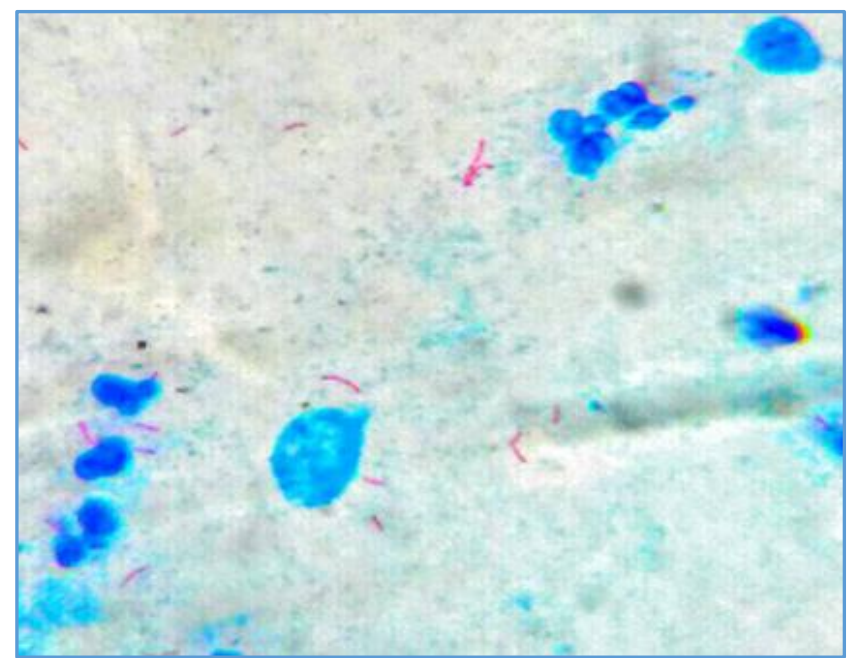

Figure 2. Acid-fast bacilli in ZN stain, ZN stain, $100 \mathrm{X}$.

\section{DISCUSSION}

In many low and middle-income countries with limited resources, the diagnosis of $\mathrm{TB}$ is still based on poorly validated symptom-based algorithms, often not resulting in a definitive diagnosis. The clinical presentation of tuberculosis is usually fever, night sweat, weight loss, anorexia. But some time delay in diagnosis has often been attributed to atypical clinical presentation and radiological presentation. The diagnosis of tuberculosis by cytomorphology is not new. It is a necrotising granulomatous infection, which cytologically demonstrates the microscopic equivalent of caseous necrosis, granular-appearing necrotic background, together with mature lymphocytes, epithelioid histiocytes and multinucleated Langhans type histiocytes. Several conditions, including mycosis, bacterial and viral adenitis can present the same cytology as Mycobacterium tubercular adenitis does. Laboratory tests may be essential to establish the cause of such adenopathy correctly, because treatment and prognosis may differ. Demonstration of Mycobacterium tuberculosis in fine needle aspirates becomes necessary for an early and accurate treatment. Fine needle aspiration cytology provides a rapid and definitive tissue diagnosis in the superficial lymphadenopathy. This study demonstrates that it also permits confirmation of the presence of mycobacteria with AFB stain in microscopy. The diagnosis of tuberculosis is confirmed by the demonstration of tubercular bacilli. Mycobacteria are slender rod shaped, non-motile, nonsporing, aerobic bacterium measuring 2 to $10 \mathrm{um}$ in length.

India has the highest TB burden as shown in the 2011 World Health Organization (WHO) statistics.[3] The diagnosis of extrapulmonary tuberculosis still remains to be more of a clinical decision. Not many clinically sensitive tests are available in India to assist the treating physician. For accurate diagnosis of M. tuberculosis, isolation and culture of organism is gold standard, but as M. tuberculosis is a slow growing organism, culture on conventional Lowenstein-Jensen medium takes 6-8 weeks. Middlebrook medium isolates growth of organism comparatively more rapidly. Mean duration to yield positive culture is about 3 weeks. But for the disease such as tuberculosis, this is too long to wait for results of culture as it is necessary to start treatment at the earliest. Therefore, comparatively rapid diagnostic strategies need to be established for diagnosis of TB lymphadenitis.[13] FNAC is a well-established diagnostic technique for lymphadenopathy evaluation. It is cost effective, safe, minimally invasive, and rapid method of diagnosing not only TB lymphadenitis but also other pathologies. It also avoids the possible physical and psychological complications of an excision biopsy.[13,14]

In this prospective study, we have examined 220 cases of lymphadenopathy referred to the Department of Pathology. The finding that the majority of the patients $(40 ; 40 \%)$ were from 11-30 years age group correlates with those of the other studies conducted by Bezabih and Mariam,[8] Lobo et al[15] Teklu et al,[16] Hart et al,[17] and Majeed and Bukhari.[18]

Most common site involved was cervical region in 75 (75\%) cases, which also correlates with the findings of other studies carried out by Bezabih et al,[8] Lau et al,[11] and Chen et al.[19] Tuberculosis was the most common finding in 50 (50\%) cases, followed by other inflammatory lymphadenitis in $50(50 \%)$. ZN stain was found to be positive for AFB in 42 $(42 \%)$ cases, which correlates with the findings of other studies conducted by Majeed and Bukhari,[18] Kheiry and Ahmed,[20] and Rajwanshi et al,[21] which reported ZN positivity of $37.4 \%, 59.4 \%$, and $40 \%$ respectively. Most common cytological pattern observed was epithelioid granuloma with caseous necrosis and with or without Langhans giant cells in 37 (37\%) cases, which is similar to the study conducted by Gupta et al.[19] Highest AFB positivity was seen in 119 (33.90\%) cases with necrosis with or without granuloma and inflammatory cells. Few cases $(54 ; 15.38 \%)$ with necrosis and granuloma showed AFB negativity whereas $36(10.25 \%)$ smears that showed necrosis and polymorphs were reported as suppurative lymphadenopathy and 105 $(29.62 \%)$ cases that did not show necrosis or granuloma and also were negative for AFB were reported as chronic nonspecific lymphadenitis, which is also similar to the study conducted by Gupta et al.[22] AFB were mostly visible in purulent aspirate whether acellular or accompanied by 
granuloma, and in the absence of ZN staining, case can be misinterpreted as an acute lymphadenitis.[23].When Culture (BACTEC) was taken as the gold standard, the sensitivity, specificity, positive predictive value (PPV), negative predictive value (NPV) and Kappa value of FNAC in the diagnosis of TB lymphadenitis results were $96.15 \%, 96.00 \%$, $95.23 \%$ and $96.77 \%$ and 0.9172 respectively. Sensitivity of FNAC was higher and diagnostic accuracy was comparable with other studies.

The diagnostic difficulties encountered were parallel to those experienced by different authors $[24,25]$ working on similar projects, a case in point being false negative cytology diagnosis in case with purulent aspirate which calls for ZN staining in every case suspected of tuberculous in origin.

\section{CONCLUSION}

Compared with AFB smear, FNAC assay has high sensitivities and cytomorphological features of FNAC on H\&E stain have significant diagnostic yield. FNAC is an optimally selected, efficient, easy to perform, and economical test for initial diagnostic workup in patients with TB lymphadenitis. Supplementation of ZN stain with FNAC increases the diagnostic yield. AFB were mostly seen in purulent aspirate whether acellular or accompanied by granuloma. In the absence of ZN staining, case can be misinterpreted as an acute lymphadenitis.

\section{REFERENCES}

[1] Croion J, Horne N, Miller F. Tuberculose clinique. UICTMR 1993:150-64.

[2] Beers MH, Porter RS, Jones TV. The Merck manual of diagnosis and therapy. 18 $8^{\text {th }}$ edn. Merck \& Co Inc 2006.

[3] TB Facts.org. TB statistics India, 2012. Available at: http://www.tbfacts.org/tb-statistics-india.html 2014.

[4] Dandapat MC, Mishra BM, Dash SP, et al. Peripheral lymph node tuberculosis: a review of 80 cases. $\mathrm{Br} \mathrm{J}$ Surg 1990;77(8):911-2.

[5] Lau SK, Kwan S, Lee J, et al. Source of tubercle bacilli in cervical lymph nodes: a prospective study. J Laryngol Otol 1991;105(7):558-61.

[6] Ng WF, Kung ITM. Clinical research pathology of tuberculous lymphadenitis: a fine needle aspiration approach. J Hong Kong Med Assoc 1990;42(1):18-21.

[7] Metre MS, Jayaram G. Acid-fast bacilli in aspiration smears from tuberculous lymph nodes. An analysis of 255 cases. Acta Cytol 1987;31(1):17-9.

[8] Bezabih M, Mariam DW, Selassie SG. Fine needle aspiration cytology of suspected tuberculous lymphadenitis. Cytopathology 2002;13(5):284-90.

[9] Singh UR, Bhatia A, Gadre DV. Cytologic diagnosis of tuberculous lymphadenitis in children by fine needle aspiration. Indian J Pediatr 1992;59(1):115-8.

[10] Shamshad SA, Shakeel A, Kafil A, et al. Study of fine needle aspiration cytology in lymphadenopathy with special reference to acid-fast staining in cases of tuberculosis. JK Science 2005;7(1):1-4.
[11] Lau SK, Wei WI, Hsu C, et al. Fine needle aspiration biopsy of tuberculous cervical lymphadenopathy. Aus N Z J Surg 1988;58(12):947-50.

[12] Dlipk DAS. Lymph nodes. In: Comprehensive cytopathology. Bibbo M, Wilbur D, (edr). $2^{\text {nd }}$ edn. Philadelphia, PA: WB Saunders 1997:707-9.

[13] Mudduwa LK, Nagahawatte AS. Diagnosis of tuberculous lymphadenitis: combining cytomorphology, microbiology and molecular techniques-a study from Sri Lanka. Indian J Pathol Microbiol 2008;51(2):195-7.

[14] Finfer M, Perchick A, Burstein DE. Fine needle aspiration biopsy diagnosis of tuberculous lymphadenitis in patients with and without the acquired immune deficiency syndrome. Acta Cytol 1991;35(3):325-32.

[15] Lobo J, Mulu G, Demmissie A. Immune response of tuberculous lymph adenitis patients to mycobacterial antigens. Abstract of 36th annual Ethiopian medical association conference, Addis Ababa, Ethiopia 2000.

[16] Teklu B, Habte D, Giday Y. Tuberculosis in children. In: Pulmonary tuberculosis: the essentials. Addis Ababa, Ethiopia: Ababa university press 1980:P 24.

[17] Hart CA, Beeching NJ, Dueren BI. Tuberculosis in the next century. J Med Microbiol 1996;44:1-34.

[18] Majeed MM, Bukhari MH. Evaluation for granulomatous inflammation on fine needle aspiration cytology using special stains. Patholog Res Int 2011;2011:8.

[19] Chen YM, Lee PY, Su WJ, et al. Lymph node tuberculosis: 7-year experience in veterans general hospital, Taipei, Taiwan. Tuber Lung Dis 1992;73(6):368-71.

[20] Kheiry J, Ahmed ME. Cervical lymphadenopathy in Khartoum. J Trop Med Hyg 1992;95(6):416-9.

[21] Rajwanshi A, Bhambhani S, Das DK. Fine needle aspiration cytology diagnosis of tuberculosis. Diagn Cytopathol 1987;3(1):13-6.

[22] Gupta AK, Nayar M, Chandra M. Critical appraisal of fine needle aspiration cytology in tuberculous lymphadenitis. Acta Cytol 1992;36(3):391-4.

[23] Pandey P, Dixit A, Mahajan NC. The diagnostic value of FNAC in assessment of superficial palpable lymph nodes: a study of 395 cases. Al Ameen J Med Sci 2013;6(4):320-7.

[24] Bandyopadhyay SN, Roy KK, Dasgupta A, et al. Role of fine needle aspiration cytology in the diagnosis of cervical lymphadenopathy. Indian Journal of Patho Microbio 1996;48(4):289-94.

[25] Agrawal P. A clinicobacteriological study of peripheral tuberculous lymphadenitis. J Asso Phy Indian 2001;49:808-12. 